\title{
Surviving Childhood Cancer: The Struggle Goes On
}

\author{
Mark A. Chesler, PhD
}

Surviving cancer is an ongoing process. Children begin the process of surviving cancer the moment the diagnosis is made. Children are surviving cancer when they are on treatment. Children are surviving cancer when they successfully complete treatment. And children are surviving cancer when they have been off treatment and free of disease symptoms for 2,5 , or 10 years or more. The definition of survivorship may be a matter of debate, but what is not debatable are the advances in medical diagnosis and treatment that mean that many more children will survive this disease than ever before. We must prepare them and ourselves for the continuing struggle ahead.

The Candlelighters Childhood Cancer Foundation has undertaken a study of psychosocial experiences and needs of long-term survivors of childhood cancer. For our purposes we have arbitrarily defined a long-term survivor as a young person with cancer who is successfully off treatment. In addition, we limited the study to young people 14 years of age and older. In this brief report some of the continuing issues these young people struggle with, and some of the assistance they desire from members of their medical treatment teams, are indicated.

\section{Common Worries}

Several recent studies of children surviving cancer delineate the issues, problems, and concerns faced by these young people. In our study of $\sim 300$ young people off treatment for cancer, they highlight seven major and common worries.

One common worry is their own physical health status. This often takes the form of concern about the possibility of a relapse or return of the disease, their physical energy level and fatigue, their sometimes diminished abilities, and their reproductive capacity (the last especially among young women).

A second major worry expressed by these survivors concerns their psychological esteem and identity. It often is difficult to decide whether to talk about cancer in the past or the present (Do I have? Did I have?) and how to (re)define oneself as no longer a patient. After all, most of these young people have adapted to the language and culture of the medical system and patient status for several years, with life and death (as well as complex treatments and pain) in the balance. Now, off treatment, a new sense of self must emerge. "Who am I now?" and "How do I present myself?" are typical questions.

A third major worry focuses on the welfare of one's immediate family. Young people surviving cancer express concern about the mental health of their parents and siblings, well aware of the special burdens and strains under which these other family members have been operating. In some cases, these concerns also involve financial issues, as they recognize that their family's budget and status may have been threatened or altered by the costs of medical treatment, transportation and lodging, etc.

A fourth major worry involves the social world of the young survivor, ie, her or his relationships with friends. Many young people report that they find it difficult to "fit in" with their old friends, and that they fear the loss of prior comrades and pals. To the extent that they may have undergone major identity changes as a result of coping with the illness, they may feel "different"-more mature, more or less socially skilled, less physically able, more in touch with what is important-from their peers. The decision about what, when, and how to tell others about the cancer is often faced by survivors as they enter or reenter new and old relationships.

From the Department of Sociology, University of Michigan, Ann Arbor, MI. (1) 1990 by Association of Pediatric Oncology Nurses. 
Dealing with the school system represents another major concern. Part of what happens in school is strictly academic. Many young people, especially those from less affluent backgrounds, report difficulty with school and in obtaining adequate assistance from school officials. Schools also are the arena within which many social and friendship relationships occur, and young people learning new forms of friendship and dating must be able to manage the school environment in order to have rewarding social lives. In addition, of course, school is the gateway to future adult achievement patterns and careers.

Future possibilities represent another major concern of long-term survivors of childhood cancer. These future concerns typically center on the availability of life and health insurance, on job and career options, and on understanding the genetic compromises that may have occurred as a result of treatment. These issues all affect a person's ability to take her or his place as a productive member of a family, an organization, and a community.

A final worry expressed by young people surviving cancer is their continuing care from a skilled and attentive medical system. Many young people leave their communities as they mature, and go elsewhere for educational or job opportunities. In so doing, they also leave behind the caring and devoted (as they report) practitioners who provided basic medical care. Who should they go to as they seek ongoing medical care? Which physicians in their new communities are prepared to understand the unique physical, psychophysical and psychosocial issues faced by survivors of childhood cancer (eg, questions about rashes or fevers that hang on, worries about minor symptoms on the anniversary of the diagnosis, etc).

\section{Services to Meet Needs and Address Concerns}

In order to competently continue and complete the care provided to children with cancer the service base must be expanded to include the needs and concerns reported by these long-term survivors. Five different kinds of services need to be provided: patient information, supportive counseling, peer support systems, community education and advocacy, and linkage to continuing medical services. These may be provided within the context of a new innovation in care: the long-term survivor clinic. Or they may be provided on a more diffuse basis, following survivors as they leave their original community and treatment facility and engage in new roles, new lives, and new systems of care.

Survivors of childhood cancer need more detailed and honest information about the long-term effects of their illness and treatment. This information must include discussion about child-bearing capacities and genetic potential (as much as is known). Young women, especially, but young men as well, often express a desire for genetic counseling and birth control information. In addition, information about the psychosocial barriers or problems noted above (eg, how to handle educational or insurance discrimination, how to manage relationships with family members and friends) would be welcome.

A second vital service is social support and in some cases supportive psychological counseling. This is not necessarily the same as psychotherapy, for it is not asked for, nor should it proceed, on the basis of an assumption of psychopathology. What is involved here are the effects on relatively normal young people of a struggle with major physical and psychological trauma over a long period of time. Personal self-esteem and identity, family relations, friendship relations, and visions of the future all may be affected by this experience. Young people often report a desire to talk about these issues with caring and experienced adults.

A third key component of an integrated service system is the availability of peer support systems. Many survivors report that they wished they had had earlier access to "other patients my age." Many hospitals and clinics are experimenting with adolescent support groups, some run by social workers or nurses and some run by young patients themselves. Obviously, the need for peer exchange and support does not end with the cessation of treatment, and the Candlelighters Foundation currently is attempting to develop national and regional networks of childhood cancer survivors that will help fill this service gap. Local medical care teams can aid in this effort by referring survivors' names and 
addresses to the Foundation, by encouraging young people to participate, and by developing local groups.

A fourth service need is the substantial expansion of public information and education campaigns. The isolation and discrimination often experienced by survivors is the result of public ignorance and fear. Efforts to educate employers, insurance agents and companies, school and civic agency personnel, and the public at large, all help create a community environment more responsive and welcoming of these young people.

Finally, linkage to continuing medical care of a sensitive and competent nature is crucial. This effort could be facilitated by the development and publication of the networks of pediatric oncology facilities and staffs that are currently active, and by sharing such information with youngsters as they make major life changes.

\title{
Conclusion
}

We all take great pleasure in the medical advances that have helped to create a growing population of survivors of childhood cancer. But we cannot permit these great gains to blind us to the work left to do, and to the continuing struggle we and survivors must undertake together.

\section{New Approaches in the Treatment of Children With Central Nervous System Tumors}

\author{
Patsy McGuire, RN, MS, CPNP
}

Cancer continues to be the leading medical cause of death in the pediatric age group younger than 15 years of age. Each year $\sim 6,000$ new cases are diagnosed and some $20 \%$ of newly diagnosed patients will have central nervous system (CNS) neoplasms. CNS neoplasms currently are the most common solid tumors in the pediatric age group with a peak incidence at diagnosis between 5 and 10 years of age.

Pediatric CNS tumors are described both histologically and by location. Common histologic groups include astrocytoma, medulloblastoma, primitive neuroectodermal tumor, ependymoma, glioma and pinealoma. Site of location is generally described as supratentorial or infratentorial.

Clinical symptoms of pediatric CNS tumors vary by the age of the child at the time of diagnosis. Symptomatology in infants tends to be somewhat nonspecific, but often includes enlarging head size, vomiting, lethargy, seizures, and often, most importantly, a loss of previously acquired motor and/or developmental milestones. In older children, symptoms may be quite specific and often include headache, visual disturbances (blurred vision, diplopia, photophobia), dizziness, ataxia, motor or sensory impairment, head tilt, personality alteration, seizures, speech disturbances, and cranial nerve dysfunction.

A variety of clinical signs are indicative of CNS tumors and should be evaluated as part of the child's

From the Children's Hospital, Denver, CO. 1990 by the Association of Pediatric Oncology Nurses. 